\title{
The gut microbiota influences skeletal muscle mass and function in mice
}

Shawon Lahiri ${ }^{1,2,}$,**, Hyejin Kim ${ }^{3}$, Isabel Garcia-Perez ${ }^{4}$, Musarrat Maisha Reza ${ }^{1,5}$, Katherine A. Martin ${ }^{3}$, Parag Kundu ${ }^{3,6}$, Laura M. Cox ${ }^{7}$, Joel Selkrig ${ }^{3, \dagger}$, Joram M. Posma ${ }^{4}$, Hongbo Zhang ${ }^{8, \S}$, Parasuraman Padmanabhan ${ }^{3}$, Catherine Moret $^{2}$, Balázs Gulyás ${ }^{9,10}$, Martin. J. Blaser ${ }^{11,12}$, Johan Auwerx ${ }^{8}$, Elaine Holmes $^{4,13}$, Jeremy Nicholson ${ }^{4,14}$, Walter Wahli ${ }^{2,3,15 !}$, and Sven Pettersson $1,3,6, ! *$

\section{Affiliations:}

${ }^{1}$ Department of Microbiology, Tumor and Cell Biology, Karolinska Institutet, Sweden.

${ }^{2}$ Center for Integrative Genomics, University of Lausanne, Lausanne, Switzerland.

${ }^{3}$ Lee Kong Chian School of Medicine, Nanyang Technological University, Singapore.

${ }^{4}$ Division of Computational and Systems Medicine, Department of Surgery and Cancer, Sir Alexander Fleming Building, Imperial College London, SW72AZ, UK.

${ }^{5}$ School of Biological Sciences, Nanyang Technological University, Singapore.

${ }^{6}$ Singapore Center for Environmental Life Sciences Engineering (SCELSE), Nanyang Technological University, Singapore.

${ }^{7}$ Ann Romney Center for Neurologic Diseases, Brigham \& Women's Hospital, Harvard Medical School, Boston, MA 02115, USA.

${ }^{8}$ Laboratory of Integrative and Systems Physiology, École Polytechnique Fédérale de Lausanne, Lausanne, Switzerland. 
${ }^{9}$ Department of Neuroscience and Mental Health, Lee Kong Chian School of Medicine, Nanyang Technological University, Singapore.

${ }^{10}$ Depertment of Clinical Neuroscience, Karolinska Institutet, Sweden.

${ }^{11}$ Department of Medicine, New York University School of Medicine, New York, NY 10016, USA.

${ }^{12}$ Medical Service, VA New York Harbor Healthcare System, New York, NY 10010, USA.

${ }^{13}$ Division of Computational and Systems Medicine, Department of Surgery and Cancer, Sir Alexander Fleming Building, Imperial College London, SW72AZ UK

${ }^{14}$ Australian National Phenome Center, Murdoch University, WA 6150

${ }^{15}$ INRA ToxAlim Integrative Toxicology and Metabolism UMR1331, Chemin de Tournefeuille, Toulouse Cedex, France.

† Current affiliation: Swedish Research Council, Sweden.

$\uparrow$ Current affiliation: European Molecular Biology Laboratory, Genome Biology Unit, Heidelberg, Germany.

$\S$ Current affiliation: Key Laboratory for Stem Cells and Tissue Engineering, Ministry of Education; The Department of Histology and Embryology of Zhongshan School of Medicine, Sun-Yat Sen University, Guangzhou, China

! Shared last authorships

*Corresponding authors 


\title{
Overline: Gut Microbiota
}

One Sentence Summary: Gut microbes transplanted into germ free mice improve skeletal muscle mass and strength.

\section{Accessible Summary}

\begin{abstract}
The functional interactions between the gut microbiota and the host are important for host physiology, homeostasis and sustained health. Germ-free mice, not exposed to microbes, displayed reduced skeletal muscle mass due to skeletal muscle atrophy when compared to pathogen-free mice that had gut microbes. Skeletal muscle of germ-free
\end{abstract}


mice display muscle atrophy, decreased expression of insulin-like-growth factor 1 (Igfl) and reduced transcription of genes associated with skeletal muscle growth and mitochondrial function. NMR spectrometry analysis of skeletal muscle, liver, and serum from germ-free mice revealed multiple changes in the amounts of amino acids, including glycine and alanine compared to the spectrum observed in mice exposed to microbes . Germ-free mice also had reduced serum choline, the precursor of acetylcholine, the key neurotransmitter between muscle and nerve at the neuromuscular junction. Reduced expression of Rapsyn and Lrp4, two proteins important for neuromuscular junction assembly and function were also observed in muscle from germ free mice. Transplanting germ-free mice with gut microbiota from pathogen-free mice, resulted in an increase in skeletal muscle mass, reduction in atrophy markers, improved oxidative metabolic capacity of muscle and elevated expression of the neuromotor junction assembly genes Rapsyn and Lrp4. Exposing germ-free mice to microbe metabolites such as short chain fatty acids, in part reversed skeletal muscle impairments. Our results suggest a role for the gut microbiota in regulating skeletal muscle mass and function in mice. 


\section{Introduction}

Skeletal muscle function is regulated by the central nervous system through neurotransmission at neuromuscular junctions (NMJs). NMJs are highly specialized chemical synapses formed between motor neurons and skeletal muscle fibers (1). Skeletal muscle displays marked plasticity being able to respond to a variety of environmental cues, such as exercise and nutrition. Skeletal muscle is also the major site of insulinstimulated glucose uptake and fatty acid oxidation emphasizing its key role in metabolism. Reduced skeletal muscle mass and function are associated with metabolic disorders (2) and sarcopenia (3), underscoring its role in maintenance of health.

It has been established that the gut microbiota influences host health in part due to its co-evolvement with the host to meet mutually beneficial biochemical and biological needs (4). There are many studies investigating how the gut microbiota influences liver and intestinal metabolism, immunity, and behavior (5-8). However, few studies have reported on how the gut microbiota regulates one of the dominant metabolic organs in the body, skeletal muscle. Here, we present evidence from germ-free and pathogen-free mice that the gut microbiota influences skeletal muscle mass and function.

\section{Results}

\section{Gut microbes modulate skeletal muscle mass in mice}

Germ-free mice (GF) that lacked gut microbiota displayed reduced skeletal muscle mass compared to pathogen-free (PF) control mice that had gut microbiota (Fig. 1A). . Transplanting germ-free mice with the gut microbiota of pathogen-free mice (henceforth mentioned as conventionalized germ-free mice, C-GF) restored muscle mass in the transplanted animals (C-GF) (Fig. 1A). Histological examination of the tibialis anterior (fast oxidative) muscle revealed a trend towards fewer but larger muscle fibers $(>2000-$ $3000 \mu^{2}$ cross-sectional fiber area) in germ-free mice compared to pathogen-free mice (Fig. S1A and B). In addition, reduced expression of the myosin heavy chain genes $M y H C I I a, I I b$, and IIx were observed in germ-free muscle compared to pathogen-free tibialis anterior muscle (Fig. 1B). A similar trend was also observed in the soleus (slow- 
oxidative) muscle and the extensor digitorum longus (fast-glycolytic) muscle of germfree mice compared to pathogen-free mice (Fig. S1C and D). Furthermore, we observed increased expression of Atrogin-1 and MuRF-1 encoding E3 ubiquitin ligases, known to be involved in muscle atrophy, in the tibialis anterior muscle of germ-free mice compared to pathogen-free mice (Fig. 1C, 1E, and 1F). Transplanting germ-free mice with the gut microbiota of pathogen-free mice reduced the expression of MuRF-1 and Atrogin-1 in anterior tibialis muscle of the C-GF mice. The expression of Atrogin-1 and MuRf-1 are known to be regulated by FoxO transcription factors (9). While elevated expression of FoxO3 expression was observed in the tibialis anterior muscle of germ-free mice, the expression was normalized in the conventionalized germ-free mice (Fig. 1C). The energy sensor AMP-activated protein kinase (AMPK) controls muscle fiber size by activating the FoxO-mediated protein degradation pathway (10). Further analysis of the tibialis anterior muscle from germ-free mice revealed an increase in phosphorylation of the catalytic domain (Thr172) of AMPK (Fig. 1E and F). It has been reported that Atrogin-1 expression regulates MyoD expression in skeletal muscle under atrophy conditions (12). We observed reduced expression of key muscle genes that regulate skeletal muscle differentiation, such as $M y o D$ and Myogenin, in germ-free skeletal muscle compared to pathogen-free skeletal muscle (Fig. 1D). Similar trends in increased expression of atrophy markers such as Atrogin-1 and MuRF-1 with a concomitant downregulation of $M y o D$ expression were also observed in soleus (slow oxidative) muscle and extensor digitorum longus (fast glycolytic) muscle of germ-free mice (Fig. S1 E-H).

Glucocorticoids are known to induce skeletal muscle atrophy under various pathological conditions (13). The transcription factor Kruppel Like Factor 15 (KLF15) is one of the target genes activated by the glucocorticoid receptor and is involved in the regulation of several metabolic processes in skeletal muscle including the upregulation of branched-chain aminotransferase 2 (BCAT2), which in turn induces the degradation of branched-chain amino acids (BCAAs) (14). A surge in corticosterone concentrations was observed in serum of germ-free mice (Fig. 2A), along with up-regulation of Klf15 expression in tibialis anterior muscle of germ-free (Fig. 2B). The observed muscle atrophy in germ-free mice was also associated with activation of the enzymes involved in the BCAA catabolic pathway. BCAAs are trans-aminated by BCAT2 to generate 
branched-chain $\alpha$-keto acids, which in turn are subjected to oxidative decarboxylation by branched-chain $\alpha$-keto acid dehydrogenase $(\mathrm{BCKDH})$ to produce Coenzyme A esters. $\mathrm{BCKDH}$ activity is negatively regulated by the BCKDH kinase (BCKDK). Increased gene expression of Bcat and Bckdh were observed in tibialis anterior muscle of germfree mice, whereas the expression of the inhibitor $B c k d k$ was reduced suggesting increased BCAA catabolism in skeletal muscle tissue of germ-free mice (Fig. 2C).

Skeletal muscle mass is maintained by the balance between protein synthesis and protein degradation. To monitor potential decline in the protein synthesis pathway, we assessed the Igf1-Akt-mTOR growth-promoting pathway. As can be seen (Fig. 2D), while the expression of the insulin-like-growth factor 1 gene (Igfl) was impaired in tibialis anterior muscle of germ-free mice, the Igf1 expression was normalized when germ-free mice were transplanted with gut microbiota of pathogen-free mice. Notably, Igf1 protein levels remain unaltered in the serum of germ-free mice (Fig. S1I), suggesting additional Igf1 regulatory loop(s), possibly involving the Igf binding proteins (IGFBPs) that are known to regulate Igfl functions. Indeed, of the six IGFBPs, we observed elevation of expression of the Igfbp 3 gene in tibialis anterior muscle of germ-free mice compared to pathogen-free mice and C-GF mice (Fig. 2D), in line with a previous observation (15). Increased expression of $I g f b p 3$ is known to exert an inhibitory effect on skeletal muscle growth suggesting on regulatory loop to explain the reduced muscle mass in skeletal muscle of germ-free mice (16). However, the activation profile of Akt, mTOR, and its downstream effector, the S6 ribosomal protein, were unaffected in muscles from germ-free mice (Fig. S1J and K).

\section{Altered metabolism in skeletal muscle of Germ-free mice}

Given that skeletal muscle of germ-free mice showed signs of atrophy, we next investigated the oxidative metabolic capacity of skeletal muscle. Histological staining revealed reduced activity of the mitochondrial enzyme succinate dehydrogenase (SDH) (Fig. 3A) and expression of the $S d h$ gene (Fig. 3B) in germ-free muscle compared to that observed in muscle from pathogen-free mice. Mitochondrial DNA content was also reduced in germ-free muscle, but was restored when transplanted with gut microbiota 
from pathogen-free mice (Fig. 3C). Additionally, we observed reduced gene expression of mitochondrial biogenesis markers, such as peroxisome proliferator-activated receptor gamma coactivator1-alpha $(P g c l \alpha)$ and mitochondrial transcription factor A (Tfam) in skeletal muscle of germ-free mice (Fig. 3D). Moreover, we noticed reduced expression of genes encoding different mitochondrial oxidative phosphorylation complexes in skeletal muscle of germ-free mice, including genes encoding cytochrome oxidase subunits of complex IV (CoxVa, CoxVIIb, and $\mathrm{CytC}$ ), known to be involved in the electron transport chain (Fig. 3E). Similar observations supporting dysfunctional mitochondrial biogenesis and oxidative capacity were also observed in two other skeletal muscle subtypes, soleus (oxidative) and extensor digitorum longus (glycolytic) muscle of germ-free mice (Fig. S2 A-D). The protein expression profile of the different oxidative phosphorylation (OXPHOS) complexes of the electron transport chain (Fig S6A) were also altered in muscle from germ-free mice. Despite a possible reduction in oxidative metabolic capacity, germ-free mice performed as well as pathogen-free mice when challenged to exercise until exhaustion (Fig. S2E).

These results prompted us to undertake further metabolic analyses of germ-free mice. Analysis of serum metabolic markers revealed reduced quantities of glucose and insulin in germ-free mice compared to pathogen-free mice (Fig. S2F). Futher investigation of metabolic pathways for fatty acid and glucose metabolism in germ-free mice muscle, revealed reduced expression of glycolytic genes encoding the enzymes phosphofructokinase $(P f k)$, pyruvate kinase $(P k)$, lactate dehydrogenase $(L d h)$, and pyruvate dehydrogenase $(P d h)$ in tibialis anterior muscle from germ-free mice. Expressions of these enzymes were restored when germ-free mice were transplanted with the gut microbiota of pathogen-free mice (Fig. 3F). A similar trend of reduced expression of glycolytic genes was also observed in the soleus and extensor digitorum longus muscles of germ-free mice (Fig. S2D and E). No differences in expression of genes encoding medium-chain acyl-CoA dehydrogenase (Mcad) and muscle specific carnitine palmitoyltransferase1b ( $m$ Cpt $1 b$ ) involved in fatty acid oxidation (Fig. $3 \mathrm{G}$ ) as well as phosphorylation of acetyl Co-A carboxylase (Fig. S2I and J) were observed in tibialis anterior muscle of germ-free mice. Cholesterol and free fatty acids in serum remained unaltered in germ-free mice compared to pathogen-free mice (Fig. S2F). Further analysis 
of glucose uptake in skeletal muscles, using FDG-PET/MRI imaging, revealed no obvious differences in glucose uptake for the back muscles and hindleg muscles of germfree when compared to pathogen-free mice (Fig. S2K and L). However, we observed an increased accumulation of glycogen in quadriceps (fast glycolytic) of muscles in germfree mice(Fig. 3H), implying possible impaired utilization of glucose by the quadriceps muscle by germ-free mice.

To further corroborate our findings, we assessed whether disruption of the gut microbiota-host environment, using antibiotics had effects on skeletal muscle. We monitored muscle samples from pathogen-free mice exposed to low-dose penicillin in order to examine consequences of disruption of host metabolic functions mediated by alterations in microbial community composition $(18,19)$. Whereas low-dose penicillin increased $\mathrm{FoxO} 3$ gene expression and reduced $\mathrm{MyoD}$ gene expression in skeletal muscle of pathogen-free mice (Fig. S3A), the expression of Atrogin-1, MuRf-1, Pgcl $\alpha$ and Tfam genes remained largely unaffected under these experimental conditions (Fig. S3B). However, electron transport chain genes (CoxVa, CoxVIIb, and CytC) were downregulated in pathogen-free mice treated with low-dose penicillin (Fig. S3C) indicating reduced oxidative metabolic capacity in skeletal muscle.

\section{Altered metabolites in skeletal muscle, liver, and serum of Germ-free mice}

To characterize the metabolic phenotype of germ-free mice in detail, proton NMR spectra were measured at $600 \mathrm{MHz}$ for skeletal muscle, liver, and serum samples from germ-free mice, pathogen-free mice and conventionalized germ-free mice with gut microbiota from pathogen-free mice (Fig. 4). Cross-validated principal component analysis and orthogonal partial least squares analysis models revealed differences in ${ }^{1} \mathrm{H}$ NMR metabolic profiles for skeletal muscle, liver, and serum from germ-free mice compared to pathogen-free mice and from germ-free mice compared to conventionalized germ-free mice transplanted with gut microbiota from pathogen-free mice (Fig. S4A, B and C). Transplanting germ-free mice with gut microbiota from pathogen-free mice normalized the metabolite profiles to that observed in pathogen-free mice. 
Our metabolic profiling identified large quantities of amino acids such as alanine and glycine in skeletal muscle of germ-free mice (Fig. 4A and Table 1). The expression of the gene encoding alanine transaminase (Alt) that results in transamination of alanine was also increased in muscle of germ-free mice (Fig. S5A). Typically, the amino acids alanine and glycine, and their carbon skeletons, enter different metabolic pathways to generate energy. However, we did not observe any change in ATP concentrations in the skeletal muscle of germ-free mice compared to the levels in pathogen-free mice and CGF mice transplanted with gut microbiota from pathogen-free mice (Fig. S5B). We next tested whether higher alanine levels in the muscle of germ-free mice served as a source for hepatic gluconeogenesis through the glucose-alanine shuttle. Reduced alanine (Fig. 3B and Table 1) along with low expression of the gene encoding glucose-6-phosphatase gene (G6Pase), a gluconeogenic marker, were observed in the liver of germ-free mice when compared to the pathogen- free mice. (Fig. S5C).

A large amount of glycine was observed in skeletal muscle of germ-free mice (Fig. 3A and Table 1). Apart from a possible increase in protein degradation of muscle tissue, glycine can be derived from intermediates of glycolysis (21). However, no changes in expression of genes encoding key enzymes of this intermediate pathway, such as phosphoglycerate dehydrogenase $(P h g d h)$ and serine hydroxymethyltransferases (Shmt1 and Shmt2) were observed in the muscle of germ-free mice (Fig. S5D). Glycine can also be generated from choline via betaine, dimethylglycine, and sarcosine. Reduced quantities of choline and dimethylglycine along with higher amounts of glycine were found in the serum of germ-free mice (Fig. 4C and Table 1). Intermediates in the choline metabolic pathway, glycerophosphocholine and betaine, were also reduced in the liver of germ-free mice (Fig. 4B and Table 1). However, no changes in expression of the genes encoding the enzymes involved in these intermediate steps---choline dehydrogenase $(C h d h)$, betaine homocysteine s-methyltransferase (Bhmt), Bhmt2, and sarcosine dehydrogenase (Sardh) -were observed in the skeletal muscle of germ-free when compared to pathogen-free mice (Fig. S5E).

The metabolite profile of liver in germ-free mice was characterized by high taurine, hypotaurine, and tauro-conjugated bile acids (taurocholic acid) affecting bile acid 
metabolism. We also observed increased dimethylamine, a product of choline degradation in the liver of germ-free mouse (Fig. 4B, Table 1). Furthermore, glycerophosphocholine, betaine (tri-methyl-glycine), and oxidized glutathione were also present in lower amounts in liver of germ-free mice, indicating potential perturbation of the cysteine metabolic pathway even though the amount of sarcosine remained high. In addition, glutamine, alanine, leucine, and valine along with glutamate were reduced in the liver of germ-free mice as compared to pathogen-free mice. Pyruvate was lower in the liver of germ-free mice as compared to pathogen-free mice. Expression profiling of genes encoding enzymes involved in mitochondrial oxidative metabolism as well as glucose metabolism revealed reduced expression of several genes involved in these pathways indicating metabolic dysfunction in liver of germ-free mice compared to that of pathogen-free mice (Fig. S5F, G).

Glycine amounts were high in serum and muscle of germ-free mice compared to pathogen-free or conventionalized mice transplanted with gut microbiota of pathogenfree mice (Fig. 4C, Table 1). However, amounts of choline, trimethylamine, and dimethylglycine were lower in serum of germ-free compared to pathogen-free and transplanted germ-free mice. In addition, acetate, pyruvate and 3-hydroxybutyrate were lower in serum of germ-free mice. We also observed differences in serum lipid profiles with high amounts of Very-Low-Density-Lipoproteins (VLDL) and low amounts of LowDensity-Lipoproteins (LDL) in germ-free mice compared to pathogen-free mice, in agreement with an earlier report (24). Overall, the metabolomics data indicates disrupted energy homeostasis in germ-free mice compared to pathogen-free mice, with a marked perturbation of the amino acid metabolic pathway (Fig. 5 4).

\section{Altered expression of Neuro-Muscular Junction (NMJ) genes in Germ-free mice}

Given that we observed low quantities of choline in the serum of GF mice (Fig. 4C, Table 1), and that choline is a substrate for the derivation of the neurotransmitter acetylcholine and essential for membrane integrity (25), we screened an array of molecules that affect NMJ development and function including acetylcholine receptors (26). We observed reduced expression of genes encoding different acetylcholine receptor subunits (Chrnal, 
Chrnb, Chrne, and Chrnd) in tibialis anterior muscle of germ-free mice(Fig. 6A) suggesting a potential impairment of assembly of acetylcholine receptors. We, therefore, monitored the expression of genes associated with formation, maturation, and maintenance of NMJs, including Rapsyn, a 43kDa receptor-associated synaptic protein (27) and the low-density lipoprotein receptor related protein 4 (Lrp4), both reported to be important for the development and assembly of acetylcholine receptors in NMJs (28). Expression of both Rapsyn and Lrp4 genes were reduced in GF mice, and this change was reversed when germ-free mice were transplanted with the gut microbiota of pathogen-free mice (Fig. 6B). Lrp4 associates with muscle-specific kinase (MuSK) to form a receptor complex necessary for agrin to bind (29). While we noted elevated expression of the MuSK gene, no change in expression of the Agrn gene, encoding its ligand agrin, was observed in muscle of germ-free mice (Fig. 6B). The reduced expression of the gene encoding troponin in skeletal musle of germ-free mice suggested possible impairment in myofiber contractility (Fig. 6C). We, therefore, evaluated muscle strength in germ-free mice. Germ-free mice displayed reduced muscle strength compared to muscle from pathogen-free mice when examined in a weights test (Fig. 6D). Germ-free mice also exhibited reduced locomotor and rearing activity compared to pathogen-free mice (Fig. 6E and F).

\section{Bacterial metabolites influence skeletal muscle mass in Germ-free mice}

Germ-free mice have elevated hypothalamic-pituitary-adrenal axis activity as shown by increased serum corticosterone (Fig. 2A). We therefore used an established in vitro cell culture model (30) to study the effects of microbial metabolites on muscle atrophy, induced by glucocorticoids. Dexamethasone treatment induced Atrogin-1 expression in differentiated $\mathrm{C} 2 \mathrm{C} 12$ myotubes in vitro (Fig. 7A). A cocktail of short chain fatty acids (SCFAs), such as those generated by microbial fermentation of dietary polysaccharides, reduced the effect of dexamethasone on Atrogin-1 expression (Fig. 7A). We also observed that SCFAs enhanced the expression of Pgcla, Tfam (Fig. 7B), CoxVa and CoxVIIb when administered to differentiated $\mathrm{C} 2 \mathrm{C} 12$ myotubes in vitro (Fig. 7C), indicating that microbial metabolites can positively regulate oxidative metabolism in 
skeletal muscle of mice under these experimental conditions. In addition, we monitored the effect of the SCFA cocktail on both basal and maximal cellular respiration. Carbonyl cyanide-4 (trifluoromethoxy) phenylhydrazone (FCCP) stimulated respiration in mitochondria by uncoupling ATP synthesis from electron transport in $\mathrm{C} 2 \mathrm{C} 12$ myotubes (Fig. S6 B and C). Under FCCP-stimulation conditions, treatment with the SCFA cocktail resulted in decreased oxygen consumption rate (Fig. S6 B) and an increased extracellular acidification rate (Fig. S6 C) in $\mathrm{C} 2 \mathrm{C} 12$ myotubes implying a metabolic switch from oxidative phosphorylation to a glycolytic mode of energy production.

We also treated GF mice in vivo with a cocktail of SCFAs. We observed a trend of increased skeletal muscle mass, particularly in the case of the gastrocnemius muscle (Fig. 7D) in germ-free mice treated with SCFAs compared to untreated germ-free mice. Furthermore, in vivo treatment of germ-free mice with SCFAs mice, reduced the expression of Atrogin-1 (Fig. 7E) although expression of MuRf-1 was higher in tibialis anterior muscle (Fig. 7E). We also noted increased expression of MyoD, a marker associated with myogenesis, in tibialis anterior muscle of SCFA-treated germ-free mice compared to untreated germ-free mice. (Fig. 7E). However, we did not observe changes in expression of genes encoding the mitochondrial transcription factors Pgcl $\alpha$ and Tfam (Fig. 7F) or electron transport chain proteins CoxVa, CoxVIIb, and CytC (Fig. 7G) in the tibialis anterior muscle of SCFA-treated germ free mice compared to untreated mice. Importantly, SCFA treatment improved muscle strength of germ-free mice in the weights test compared to untreated GF mice (Fig. 7H). No alterations in gene expression of the NMJ markers, Rapsyn and MuSK, were observed in tibialis anterior muscle of SCFAtreated mice compared to untreated GF mice (Fig. 7I).

\section{Discussion}

Here we show that the gut microbiota contributes to modulate skeletal muscle mass and function in mice. Germ-free mice displayed reduced muscle mass and signs of muscle atrophy through increased expression of FoxO, Atrogin-1, Murf-1 and MyoD along with with reduced muscle strength. Activation of AMPK in germ-free mouse muscle further suggests that the AMPK-FoxO3-atrogenes cascade could be one possible signaling 
pathway to explain, at least in part, the atrophy observed in germ-free muscle tissues. Similar elevation in AMPK phosphorylation in gastrocnemius muscle of germ-free mice fed on a Western diet for 5 weeks was previously reported (11), but not from mice in the normal physiological setting as shown in our study. In addition, our results suggest alteration in IGF1 protein expression locally in the muscle of germ-free mice, rather than peripheral effects of circulating IGF-1. The Akt-mTOR pathway appeared to be unaffected in muscle of germ-free mice. This suggests that protein degradation exceeded protein synthesis, which in part could contribute to the reduced skeletal muscle mass observed in germ-free mice. Our observations of increased corticosterone concentrations in serum, induction of Klf15 gene expression and activation of the BCAA pathway indicate a possible HPA-glucocorticoid driven atrophy of skeletal muscle mass in germfree mice. Skeletal muscle atrophy observed in mice maintained under germ-free conditions, thus, might be the consequence of dysregulation in several signaling pathways controlling skeletal muscle mass. The reduced levels of choline in serum, along with marked changes of gene expression of NMJ linked proteins in muscle of germ-free mice, defective locomotion and grip strength capacity, are therefore possible additional factors affecting muscle mass and function in germ-free mice. Furthermore, our observations from muscles of different metabolic subtypes suggest that the phenotypic changes observed in germ-free mice are not unique to muscle with specific metabolic characteristic but have a consistent impact on skeletal muscle as a consequence of the absence of gut microbiota.

Gut microbes synthesize amino acids and make them available to the host (35). Analyses of skeletal muscle, liver, and serum metabolites revealed altered amino acid metabolism in GF mice. Glycine and alanine were increased in GF compared to PF mouse skeletal muscle. Glycine has important implications in skeletal muscle wasting (36), acts as an energy source for muscle (37) and is also induced in response to mitochondrial stress (22). BCAA catabolism is associated with skeletal muscle protein breakdown and correlated with low leucine and valine concentrations in liver tissue and serum of germ-free mice. Decreased quantities of BCAAs, as seen in liver and serum of germ-free mice, have been associated with severe catabolic conditions, such as cachexia in terminal stages of cancer $(38,39)$. BCAAs not only serve as an alternate energy 
substrate but also are known to improve nitrogen retention and protein synthesis (40). They represent the major nitrogen source for alanine synthesis in muscle. Prior to their usage as fuel and being fed into the tricarboxylic acid cycle, BCAAs undergo a series of trans-amination steps eventually resulting in production of alanine. High quantities of alanine along with increased Alt gene expression observed in msucle of germ-free mice might thus be a consequence of muscle protein breakdown resulting in increased bioavailability of amino acids to cope with the stress generated due to the absence of gut microbiota.

Many interactions between the host and its gut microbiota are mediated by SCFAs generated from the bacterial fermentation of dietary polysaccharides (31-33). From our observations, SCFAs support, in part, skeletal muscle function by preventing atrophy and increasing muscular strength. However, the diverse biosynthetic activity of the gut microbiota beyond providing SCFAs to be used by the host as an energy source may explain why only SCFAs supplementation in germ-free mice could not fully rescue the muscle impairment phenotype. Microbes and their metabolites are likely to engage multiple converging pathways to regulate host muscle growth and function. Additional microbial products other than SCFAs might also be involved in skeletal muscle growth and function. Our study has elucidated several underlying molecular mechanisms supporting regulation of skeletal muscle mass and function in germ-free mice. While, germ-free mice, an animal model used to study microbe-host interactions is an artificial system, these mice, devoid of exposure to living microbes, provide opportunities to study microbe-host interactions in vivo. Deficits in physiological and metabolic processes due to maintaining mice under germ-free conditions are well documented. The use of low dose antibiotic treatment helped us to support our observations in germ-free mice that implied the lack of gut microbiota in reduced muscle mass and function. However, the muscle tissue samples collected from the antibiotic experiment was obtained from pathogen-free mice raised in a different animal facility and hence, should be interpreted cautiously. While our observations also suggest that the communication between muscle cells and nerve cells via the NMJs may be impaired in germ-free condition, further experiments, including physiological experiments are required to reach definitive conclusion. Additional studies are also warranted to identify specific bacterial produced 
metabolites or other microbial products to influence skeletal muscle growth and function. In conclusion, we have demonstrated the existence of a gut microbiota-skeletal muscle axis that opens for further mechanistic and physiological studies for a better understanding of mechanisms regulating this highly important metabolic organ by the gut microbiota. 


\section{Materials and Methods}

\section{Study design}

The objective of this study was to elucidate the functional interactions between the gut microbiota and the host in the regulation of skeletal muscle mass and function. Muscle was assessed in germ-free mice and pathogen-free mice maintained on autoclaved R36 Lactamin chow (Lactamin), provided with sterile drinking water ad libitum, and kept under 12-h light/dark cycles in state-of-art germ-free animal housing facilities. Skeletal muscle mass was examined and differences in expression of genes encoding different cell signaling molecules that regulate muscle mass were characterized. Detailed metabolic characterization was also performed using NMR spectrometric analyses of skeletal muscle, liver and serum samples. The influence of the gut microbiota on NMJs was also examined by analyzing expression of $\mathrm{NMJ}$ proteins and testing mouse muscle strength in the grip strength test in germ-free mice compared to pathogen-free mice. The gut microbiota's role in muscle mass and function was further analysed by transplanting germ-free mice with the gut microbiota of pathogen-free animals or treating them with microbial metabolites (short chain fatty acids). We examined the effects alterations of gut microbiota. composition on muscle by treating pathogen-free mice with low-dose penicillin. Three independent cohorts of pathogen-free, germ-free and conventionalized germ-free groups have been used with a minimum of 5 mice included in each experimental group. Blinding of researchers about the knowledge of group allocation has been ensured along with randomization of the groups to limit the occurrence of conscious and unconscious bias during experimentation. .

\section{Animals}

Germ-free and pathogen-free C57BL/6J male mice (6 to 8 weeks old) were raised in the Core Facility for Germ-Free Research, Karolinska Institutet, Sweden, and LKC School of Medicine GF research facility, Singapore, in accordance with the regulatory standards of each institution. All experiments were approved by respective local ethical committees. Germ-free mice were raised in special sterile isolators. Isolator sterility was analyzed weekly by plating fecal homogenates onto different types of agar plates to detect aerobic and anaerobic bacteria and fungi. To transplant germ-free with gut microbiota of 
pathogen-free mice, germ-free mice were conventionalized (C-GF) post-weaning by gavage of $100 \mu 1$ of homogenate generated by dissolving two fecal pellets from pathogenfree mice in $1 \mathrm{ml}$ of PBS. After gavage, C-GF mice were co-housed with pathogen-free mice for 21 days before they were sacrificed. The control group was gavaged with sterile PBS. Mice were sacrificed under isoflurane anesthesia. All animals were maintained on autoclaved R36 Lactamin chow (Lactamin), provided with sterile drinking water ad libitum, and kept under 12-h light/dark cycles. Three independent cohorts of pathogenfree, germ-free and germ-free mice transplanted with gut microbiota of pathogen-free mice were used with a minimum of 5 mice in each group. The behavioral assays were conducted in accordance with national guidelines for the care and use of laboratory animals for scientific purposes, with approved protocols from the Institutional Animal Care and Use Committees of Duke-NUS Graduate Medical School, Singapore.

SCFA treatment of germ-free mice. Germ-free mice (6-8 weeks of age) were treated with a cocktail of SCFA as per a previously published protocol (47). Briefly, mice ( $\mathrm{n}=6$ per group) were fed a mix of SCFA $(67.5 \mathrm{mM}$ sodium acetate, $40 \mathrm{mM}$ sodium butyrate, $25.9 \mathrm{mM}$ sodium propionate) in their drinking water for a period of 4 weeks. No decrease in water intake was noted, and mice were monitored for signs of dehydration. Sterility of the GF and SCFA-treated GF cohort was assessed twice a week and maintained throughout this study. After 4 weeks, mice were euthanized by inhalation of isoflurane and sacrificed, and muscles were harvested. TA muscles were used for qPCR analyses.

Antibiotic treatment.C57BL/6J mice were given low-dose penicillin $(1 \mu \mathrm{g} / \mathrm{g}$ body weight, LDP) via drinking water or no antibiotics (control) for 4 weeks postweaning as previously reported (18).

\section{Weights test}

Weight tests were performed to measure muscular strength as described previously (41).. Briefly, each mouse was held by the middle of the tail and slowly lowered to grasp the first weight (26 g) in a well-lit fume hood. Upon grasping the wire scale, mice were raised until the weight was fully raised above the bench. The criterion was met if the mouse could hold the weight for $3 \mathrm{~s}$. If the weight was dropped in before $3 \mathrm{~s}$, the time was noted, and the 
mouse rested for $10 \mathrm{~s}$ before performing a repeat trial. Each mouse was allowed a maximum of 5 trials before being assigned the maximum time achieved; if it successfully held the weight for $3 \mathrm{~s}$, it was allowed to progress to the next heaviest weight. The apparatus comprised 6 weights, weighing 26g, 33g, 44g, 63g, 82g, and 100g. All cage mates of each group were tested using a given weight before progressing to the next-heaviest weight. The total score for each mouse was then calculated as the product of the number of links in the heaviest chain held for the full $3 \mathrm{~s}$, multiplied by the time (s) it was held.

\section{Activity in the open field task}

Locomotor and rearing activities were monitored for individual mice using an automated Omnitech Digiscan apparatus (AccuScan Instruments, Columbus, OH, USA), as described previously (42). Mice were monitored in the open field for $60 \mathrm{~min}$. Locomotion was measured as total distance traveled and rearing as vertical activity.

\section{Treadmill running task}

Eight-week-old mice were subjected to exercise on a treadmill (Columbus, OH, USA) using a previously reported exercise regimen (43). Mice in all groups were acclimated to moderate treadmill running $(10 \mathrm{~m} / \mathrm{min}$ for $15 \mathrm{~min}$; 5 incline) for 2 days. After acclimation, for the exercise test, mice ran on a treadmill set at a gradually increasing speed from 0 to $15 \mathrm{~m} / \mathrm{min}$ and then maintained at a constant speed until exhaustion. Mice were removed from the treadmill upon exhaustion and had free access to food and water after the running protocol was completed. Mice were sacrificed $3 \mathrm{~h}$ after the exercise test.

\section{PET-MRI}

Mice were fasted for $4 \mathrm{~h}$ prior to the imaging session. They were imaged under anesthesia using a nanoScan PET/MRI scanner (Mediso, Hungary) under anesthesia (44). The whole body MRI images were acquired with the 1 T MRI component of the nanoScan PET/MRI scanner. All images and data analyses for the FDG PET images were performed using PMOD (version 3.5; PMOD Technologies). 
PET acquisition: A 40 min long 3D static PET scan was performed after IV injection of FDG; the animals were moving freely during the waiting period. The injected radioactivity was $10.0-15 \mathrm{MBq}$, and the maximum injected volume was $0.1 \mathrm{ml}$.

MRI acquisition. A 35-mm mouse whole body coil was used for the MRI scan. $0.8 \mathrm{~mm}$ slices were obtained using a 2D T1 SD coronal sequence with 100-mm square FOV, 256 $\times 256$ matrix, 1034-ms repetition time (TR), 12.4-ms echo time (TE), and 90 degree flip angle.

Image analysis: The PET images were first automatically registered to the MRI images using the FUSION tool in PMOD. The MRI images were then used for the manual delineation of volumes of interest (VOI). VOIs for hind leg muscle and back muscle were used for the analysis.

\section{Cell culture and treatments}

Murine $\mathrm{C} 2 \mathrm{C} 12$ myoblasts (ATCC, USA) were cultured and were considered myotubes after $96 \mathrm{~h}$ of differentiation. Differentiated cells were then treated with $1 \mathrm{mM}$ dexamethasone (Dex, Sigma-Aldrich, USA) for 24h, along with a cocktail of SCFAs, such as acetate, propionate, and butyrate, based on the molar ratio of their availability in the plasma (60:25:15 for acetate: propionate: butyrate) (45).

\section{In vitro cellular respiration assay.}

Oxygen consumption in differentiated $\mathrm{C} 2 \mathrm{C} 12$ cells in response to bacterial metabolites (i.e., SCFAs) was measured using XF96 equipment (Seahorse Bioscience), as described previously (46).

\section{Metabolomic analyses of skeletal muscle, liver, and serum.}

${ }^{1} \mathrm{H}$ NMR spectroscopy was performed on the aqueous-phase extracts from skeletal muscle (gastrocnemius), liver, and serum at $300 \mathrm{~K}$ on a Bruker $600 \mathrm{MHz}$ spectrometer (Bruker Biospin, Karlsruhe, Germany) as described in (48). Metabolic network was 
generated using the freely available MetaboNetworks software (49).

Sample treatment. Hydrophilic metabolites were extracted from liver and skeletal muscle (gastrocnemius). Samples were homogenized (64,000 rpm, $2 \mathrm{~min}$ ) in $7.5 \mathrm{ml}$ chloroform:methanol (2:1). The homogenate was combined with $1 \mathrm{ml}$ water, mixed by vortexing, and centrifuged at $13,000 \times g$ for $5 \mathrm{~min}$. The upper aqueous phase was separated from the lower organic phase and dried using nitrogen gas. Frozen serum samples $\left(-80^{\circ} \mathrm{C}\right)$ were thawed and then centrifuged at $2700 \times \mathrm{g}$ for $10 \mathrm{~min}$ to remove particulates and precipitated proteins. $300 \mu \mathrm{l}$ of individual serum samples was prepared with $\mathrm{pH} 7.4$ phosphate buffer, as described previously for high resolution ${ }^{1} \mathrm{H}$ NMR spectroscopy (48).

${ }^{1} \mathbf{H}$ NMR metabolic profiling analysis. The hydrophilic phase of liver and skeletal muscle was reconstituted in $540 \mu \mathrm{l}$ of $\mathrm{D}_{2} \mathrm{O}$ and $60 \mu \mathrm{l}$ of phosphate buffer $(\mathrm{pH} 7.4,80 \%$ $\mathrm{D}_{2} \mathrm{O}$ ) containing $1 \mathrm{mM}$ of the internal standard, 3-(trimethylsilyl)-[2,2,3,3,-2H4]propionic acid (TSP). ${ }^{1} \mathrm{H}$ NMR spectroscopy was performed on the aquose phase extracts at $300 \mathrm{~K}$ on a Bruker $600 \mathrm{MHz}$ spectrometer (Bruker Biospin, Karlsruhe, Germany) using the following standard one-dimensional pulse sequence with saturation of the water resonance: relaxation delay $(\mathrm{RD})-\mathrm{gz}, 1-90^{\circ}-\mathrm{t}-90^{\circ}-\mathrm{tm}-\mathrm{gz}, 2-90^{\circ}-$ acquisition time (ACQ), where $\mathrm{RD}$ is the relaxation delay, $90^{\circ}$ represents the applied $90^{\circ}$ radio frequency (RF) pulse, $\mathrm{t} 1$ is an inter-pulse delay set to a fixed interval of $4 \mu \mathrm{s}$; $\mathrm{RD}$ was $2 \mathrm{~s}$ and tm (mixing time) was $100 \mathrm{~ms}$ (48). Water suppression was achieved through irradiation of the water signal during $\mathrm{RD}$ and $\mathrm{tm}$. For the the liver and muscle samples, each spectrum was acquired using 4 dummy scans followed by 32 scans and collected into $64 \mathrm{~K}$ data points. A spectral width of $20,000 \mathrm{~Hz}$ was used for all samples. Prior to Fourier transformation, the Free Induction Decay (FIDs) were multiplied by an exponential function corresponding to a line broadening of $0.3 \mathrm{~Hz} .{ }^{1} \mathrm{H}$ NMR spectra were manually corrected for phase and baseline distortions and referenced to the TSP singlet at $\delta$ 0.0. Spectra were digitized using an in-house MATLAB (version R2014a, The Mathworks, Inc.; Natwick, MA, USA) script. Spectra were subsequently referenced to the internal chemical shift reference (trimethylsilyl-[2,2,3,3,-2H4]-propionate, TSP) at $\delta$ 
0.0. Spectral regions corresponding to the internal standard ( $\delta-0.5$ to 0.5$)$ and water $(\delta$ 4.5 to 5.5$)$ were excluded.

The serum samples were analyzed using ${ }^{1} \mathrm{H}$ NMR spectroscopy with two different pulse sequences (48). The first, the so-called noesy preset sequence, provides an overview of all proton-containing species and yields sharp peaks for small molecule species, broad bands from the lipoproteins (used later for lipoprofile analysis, see below), and a broad largely featureless background from proteins, the most abundant being albumin. The second pulse sequence, the so-called Carr-Purcell-Meiboom-Gill (CPMG) sequence, takes advantage of the different nuclear spin relaxation times between large and small molecules to attenuate the peaks from large molecules (those with shorter spinspin relaxation times) to leave mainly small molecule metabolite peaks. The latter have been used for the main analysis. Experiments were performed using a standard $1 \mathrm{D}{ }^{1} \mathrm{H}$

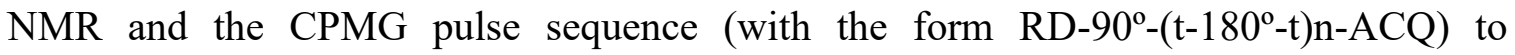
attenuate peaks from larger and more slowly moving molecules (such as lipoproteins and lipids) with a view to identifying low-molecular-weight metabolites. A RD of $4 \mathrm{~s}$, a mixing time of $0.01 \mathrm{~s}$, a spin-echo delay of $0.3 \mathrm{~ms}, 128$ loops, and an FID ACQ of 3.067 s were applied. A total of 32 scans were recorded into $73 \mathrm{k}$ data points with a spectral width of $20 \mathrm{ppm}$. Each spectrum was normalized to Probabilistic Quotient Normalization. Multivariate statistical analysis based on pattern recognition techniques such as principal component analysis and orthogonal partial least squares discriminant analysis (OPLSDA) was performed with Pareto scaling in SimcaP+14 applied to all data variables. OPLS-DA was used to compare independently each data set of ${ }^{1} \mathrm{H}$ NMR metabolic profiles. The robustness of the models was evaluated based on $\mathrm{R}^{2}$ (explained variance) and $\mathrm{Q}^{2}$ (capability of prediction) values as well as sevenfold cross-validation and class permutation validation.

Metabolite identifications. Confirmation of significant NMR metabolite identities was obtained using 1D and 2D NMR experiments (spike-in of chemical standards, J-resolved spectroscopy (known as JRES), TOtal Correlation SpectroscopY (known as TOCSY), Hetero-nuclear Single Quantum Coherence (known as HSQC) spectroscopy).

\section{Histological assessment of skeletal muscle fiber morphology and oxidative capacity.}


For histological staining, serial cross-sections $(10 \mu \mathrm{m})$ were cut from the mid-belly of the TA muscle, fixed in O.C.T and frozen in isopentane cooled by liquid nitrogen. Hematoxylin and eosin ( $\mathrm{H} \& \mathrm{E}$ ) staining was used for muscle fiber cross-sectional area measurements. The histochemical assay for SDH activity on the TA muscle sections was used to distinguish between oxidative and non-oxidative fibers, as described previously (50).

\section{Glycogen measurements in muscle.}

Glycogen content was estimated in quadriceps muscle tissues using a glycogen assay kit (Biovision, USA) and following the manufacturer's instructions.

\section{Serum analyses.}

Serum was prepared from blood collected and stored at $-70^{\circ} \mathrm{C}$ until assayed using the Roche/Hitachi 902 robot system (Roche Diagnostics, Rotkreutz, Switzerland) following the manufacturer's instructions.

\section{Corticosterone estimation.}

Corticosterone was estimated in serum using the Corticosterone ELISA kit (Abcam) following the manufacturer's instructions.

\section{Gene expression analysis by real-time quantitative RT-PCR (qRT-PCR).}

Total RNA was extracted from cells and muscle tissues using Trizol reagent following the manufacturer's instructions. For SYBR-Green-Based qRT-PCR, isolated RNA was reverse-transcribed using SuperScript II and random primers (Invitrogen). qRT-PCR assays were performed in triplicate, and data were normalized to Rpl27 and Rpl13 and analyzed using qBase software. Details regarding gene-specific primers are provided in Supplemental Table S1.

\section{Mitochondrial DNA copy number quantification.}

The relative copy number of mitochondrial DNA (mtDNA) per nuclear DNA (nuDNA) ratio was measured by qPCR.

\section{Western blot analysis.}


Tissue extracts $(50 \mu \mathrm{g} / \mathrm{lane})$ were prepared in lysis buffer $[10 \mathrm{mM}$ Tris- $\mathrm{HCl}(\mathrm{pH} 8), 150$ $\mathrm{mM} \mathrm{NaCl}, 1 \%$ Triton X-100, and protease inhibitors and PhosSTOP ${ }^{\mathrm{TM}}$ (Roche)] and subjected to western blotting. The western blot was probed with anti-phospho-AMPK and anti-AMPK, p-ACC and ACC, p-mTOR and mTOR, p-S6 and S6 (Cell Signaling), and Tubulin antibodies. Immunodetection with an appropriate secondary peroxidaseconjugated antibody (DAKO) was followed by chemiluminescence (ECL; Amersham). Protein band quantification was performed using LabImage software.

\section{Statistical analysis.}

Statistical significance was determined using GraphPad Prism 7 statistical software. For all experiments that required analyses between multiple groups (PF, GF and C-GF), Oneway analysis of variance (ANOVA) followed by Tukey's post hoc test was performed. Two-tailed Student's t-test was performed when observations between only two groups were compared. F-test was performed prior to Student's t-test to determine equal variance between the two groups. $\mathrm{P}<0.05$ was considered statistically significant unless otherwise stated. Values are expressed as mean \pm SEM.

\section{Supplementary Materials}

Fig. S1. The gut microbiota affects skeletal muscle function in mice.

Fig. S2. The gut microbiota influences skeletal muscle oxidative capacity in mice.

Fig. S3. A subtherapeutic dose of antibiotics affects skeletal muscle mass and function in mice.

Fig. S4. Metabolomic analyses in mouse muscle, liver and serum.

Fig. S5. Effect of the gut microbiota on metabolic pathways in mice.

Fig. S6. Short chain fatty acids (SCFAs) influence oxidative capacity of mouse skeletal muscle.

Table S1. List of primer sequences. 


\section{References}

1. L. A. Tintignac, H.-R. Brenner, M. A. Rüegg, Mechanisms Regulating Neuromuscular Junction Development and Function and Causes of Muscle Wasting, Physiol. Rev. 95, 809-852 (2015).

2. D. E. Kelley, B. Goodpaster, R. R. Wing, J. A. Simoneau, Skeletal muscle fatty acid metabolism in association with insulin resistance, obesity, and weight loss, Am. J. Physiol. 277, E1130-E1141 (1999).

3. K. S. Nair, Aging muscle, Am. J. Clin. Nutr. 81, 953-963 (2005).

4. J. C. Clemente, L. K. Ursell, L. W. Parfrey, R. Knight, The impact of the gut microbiota on human health: an integrative view, Cell 148, 1258-1270 (2012).

5. P. S. Kabouridis, R. Lasrado, S. McCallum, S. H. Chng, H. J. Snippert, H. Clevers, S. Pettersson, V. Pachnis, Microbiota controls the homeostasis of glial cells in the gut lamina propria, Neuron 85, 289-295 (2015).

6. V. Braniste, M. Al-Asmakh, C. Kowal, F. Anuar, A. Abbaspour, M. Tóth, A. Korecka, N. Bakocevic, L. G. Ng, N. L. Guan, P. Kundu, B. Gulyás, C. Halldin, K. Hultenby, H. Nilsson, H. Hebert, B. T. Volpe, B. Diamond, S. Pettersson, The gut microbiota influences blood-brain barrier permeability in mice, Sci. Transl. Med. 6, 263ra158 (2014).

7. R. Diaz Heijtz, S. Wang, F. Anuar, Y. Qian, B. Björkholm, A. Samuelsson, M. L. Hibberd, H. Forssberg, S. Pettersson, Normal gut microbiota modulates brain development and behavior, Proc. Natl. Acad. Sci. U. S. A. 108, 3047-3052 (2011).

8. A. Montagner, A. Korecka, A. Polizzi, Y. Lippi, Y. Blum, C. Canlet, M. TremblayFranco, A. Gautier-Stein, R. Burcelin, Y. C. Yen, H. S. Je, A. A. Maha, G. Mithieux, V. Arulampalam, S. Lagarrigue, H. Guillou, S. Pettersson, W. Wahli, Hepatic circadian clock oscillators and nuclear receptors integrate microbiome-derived signals, Sci. Rep. 
16, 20127 (2016).

9. M. Sandri, C. Sandri, A. Gilbert, C. Skurk, E. Calabria, A. Picard, K. Walsh, S. Schiaffino, S. H. Lecker, A. L. Goldberg, Foxo transcription factors induce the atrophyrelated ubiquitin ligase atrogin-1 and cause skeletal muscle atrophy, Cell 117, 399-412 (2004).

10. E. L. Greer, P. R. Oskoui, M. R. Banko, J. M. Maniar, M. P. Gygi, S. P. Gygi, A. Brunet, The energy sensor AMP-activated protein kinase directly regulates the mammalian FOXO3 transcription factor, J. Biol. Chem. 282, 30107-30119 (2007).

11. F. Bäckhed, J. K. Manchester, C. F. Semenkovich, J. I. Gordon, Mechanisms underlying the resistance to diet-induced obesity in germ-free mice, Proc. Natl. Acad. Sci. U. S. A. 104, 979-984 (2007).

12. L. A. Tintignac, J. Lagirand, S. Batonnet, V. Sirri, M. P. Leibovitch, S. A. Leibovitch, Degradation of MyoD mediated by the SCF (MAFbx) ubiquitin ligase, J. Biol. Chem. 280, 2847-2856 (2005).

13. S. H. Lecker, V. Solomon, W. E. Mitch, A. L. Goldberg, Muscle protein breakdown and the critical role of the ubiquitin-proteasome pathway in normal and disease states, $J$. Nutr. 129, 227S-237S (1999).

14. D. S. Waddell, L. M. Baehr, J. van den Brandt, S. A. Johnsen, H. M. Reichardt, J. D. Furlow, S. C. Bodine, The glucocorticoid receptor and FOXO1 synergistically activate the skeletal muscle atrophy-associated MuRF1 gene, AJP Endocrinol. Metab. 295, E78597 (2008).

15. M. Schwarzer, K. Makki, G. Storelli, I. Machuca-Gayet, D. Srutkova, P. Hermanova, M. E. Martino, S. Balmand, T. Hudcovic, A. Heddi, J. Rieusset, H. Kozakova, H. Vidal, F. Leulier, Lactobacillus plantarum strain maintains growth of infant mice during chronic undernutrition, Science 351, 854-857 (2016).

16. T. Modric, J. V Silha, Z. Shi, Y. Gui, A. Suwanichkul, S. K. Durham, D. R. Powell, L. J. Murphy, Phenotypic manifestations of insulin-like growth factor-binding protein-3 overexpression in transgenic mice, Endocrinology 142, 1958-1967 (2001). 
17. N. Suárez-Zamorano, S. Fabbiano, C. Chevalier, O. Stojanović, D. J. Colin, A. Stevanović, C. Veyrat-Durebex, V. Tarallo, D. Rigo, S. Germain, M. Ilievska, X. Montet, Y. Seimbille, S. Hapfelmeier, M. Trajkovski, Microbiota depletion promotes browning of white adipose tissue and reduces obesity, Nat. Med. 21, 1497-14501 (2015).

18. L. M. Cox, S. Yamanishi, J. Sohn, A. V Alekseyenko, J. M. Leung, I. Cho, S. G. Kim, H. Li, Z. Gao, D. Mahana, J. G. Zárate Rodriguez, A. B. Rogers, N. Robine, P. Loke, M. J. Blaser, Altering the intestinal microbiota during a critical developmental window has lasting metabolic consequences, Cell 158, 705-721 (2014).

19. I. Cho, S. Yamanishi, L. Cox, B. A. Methé, J. Zavadil, K. Li, Z. Gao, D. Mahana, K. Raju, I. Teitler, H. Li, A. V Alekseyenko, M. J. Blaser, Antibiotics in early life alter the murine colonic microbiome and adiposity, Nature 488, 621-626 (2012).

20. R. Manickam, H. Oh, C. Tan, E. Paramalingam, W. Wahli, Metronidazole Causes Skeletal Muscle Atrophy and Modulates Muscle Chronometabolism, Int. J. Mol. Sci. 19, 2418-2432 (2018).

21. M. Jain, R. Nilsson, S. Sharma, N. Madhusudhan, T. Kitami, A. L. Souza, R. Kafri, M. W. Kirschner, C. B. Clish, V. K. Mootha, Metabolite Profiling Identifies a Key Role for Glycine in Rapid Cancer Cell Proliferation, Science 336, 1040-1044 (2012).

22. P. M. Quirós, M. A. Prado, N. Zamboni, D. D’Amico, R. W. Williams, D. Finley, S. P. Gygi, J. Auwerx, Multi-omics analysis identifies ATF4 as a key regulator of the mitochondrial stress response in mammals, J. Cell Biol. 216, 2027-2045 (2017).

23. S. P. Claus, T. M. Tsang, Y. Wang, O. Cloarec, E. Skordi, F.-P. Martin, S. Rezzi, A. Ross, S. Kochhar, E. Holmes, J. K. Nicholson, Systemic multicompartmental effects of the gut microbiome on mouse metabolic phenotypes, Mol. Syst. Biol. 4, 219 (2008).

24. V. R. Velagapudi, R. Hezaveh, C. S. Reigstad, P. Gopalacharyulu, L. Yetukuri, S. Islam, J. Felin, R. Perkins, J. Boren, M. Oresic, F. Backhed, The gut microbiota modulates host energy and lipid metabolism in mice, J. Lipid Res. 51, 1101-1112 (2010).

25. R. S. Jope, D. J. Jenden, The utilization of choline and acetyl coenzyme A for the synthesis of acetylcholine, J. Neurochem. 35, 318-325 (1980). 
26. M. Akaaboune, S. M. Culican, S. G. Turney, J. W. Lichtman, Rapid and reversible effects of activity on acetylcholine receptor density at the neuromuscular junction in vivo, Science 286, 503-507 (1999).

27. M. Gautam, P. G. Noakes, J. Mudd, M. Nichol, G. C. Chu, J. R. Sanes, J. P. Merlie, Failure of postsynaptic specialization to develop at neuromuscular junctions of rapsyndeficient mice, Nature 377, 232-236 (1995).

28. S. D. Weatherbee, K. V Anderson, L. A. Niswander, LDL-receptor-related protein 4 is crucial for formation of the neuromuscular junction, Development 133, 4993-5000 (2006).

29. N. Kim, A. L. Stiegler, T. O. Cameron, P. T. Hallock, A. M. Gomez, J. H. Huang, S. R. Hubbard, M. L. Dustin, S. J. Burden, Lrp4 is a receptor for Agrin and forms a complex with MuSK, Cell 135, 334-342 (2008).

30. M. Menconi, P. Gonnella, V. Petkova, S. Lecker, P.-O. Hasselgren, Dexamethasone and corticosterone induce similar, but not identical, muscle wasting responses in cultured L6 and C2C12 myotubes, J. Cell. Biochem. 105, 353-364 (2008).

31. D. R. Donohoe, N. Garge, X. Zhang, W. Sun, T. M. O'Connell, M. K. Bunger, S. J. Bultman, The microbiome and butyrate regulate energy metabolism and autophagy in the mammalian colon, Cell Metab. 13, 517-526 (2011).

32. D. R. Donohoe, A. Wali, B. P. Brylawski, S. J. Bultman, S. Bereswill, Ed. Microbial regulation of glucose metabolism and cell-cycle progression in mammalian colonocytes, PLoS One 7, e46589 (2012).

33. D. L. Sewell, B. S. Wostmann, C. Gairola, M. I. Aleem, Oxidative energy metabolism in germ-free and conventional rat liver mitochondria, Am. J. Physiol. 228, 526-529 (1975).

34. Y. Li, Y. il Lee, W. J. Thompson, Changes in aging mouse neuromuscular junctions are explained by degeneration and regeneration of muscle fiber segments at the synapse, J. Neurosci. 31, 14910-14919 (2011).

35. C. C. Metges, Contribution of microbial amino acids to amino acid homeostasis of the 
host, J. Nutr. 130, 1857S-1864S (2000).

36. D. J. Ham, K. T. Murphy, A. Chee, G. S. Lynch, R. Koopman, Glycine administration attenuates skeletal muscle wasting in a mouse model of cancer cachexia., Clin. Nutr. 33, 448-458 (2014).

37. E. Meléndez-Hevia, P. De Paz-Lugo, A. Cornish-Bowden, M. L. Cárdenas, A weak link in metabolism: the metabolic capacity for glycine biosynthesis does not satisfy the need for collagen synthesis, J. Biosci. 34, 853-872 (2009).

38. K. Fearon, J. Arends, V. Baracos, Understanding the mechanisms and treatment options in cancer cachexia, Nat. Rev. Clin. Oncol. 10, 90-99 (2012).

39. S. H. Adams, Emerging perspectives on essential amino acid metabolism in obesity and the insulin-resistant state, Adv. Nutr. 2, 445-456 (2011).

40. P. L. de Campos-Ferraz, I. Andrade, W. das Neves, I. Hangai, C. R. R. Alves, A. H. Lancha Jr, An overview of amines as nutritional supplements to counteract cancer cachexia, J. Cachexia. Sarcopenia Muscle 5, 105-110 (2014).

41. P. Wong, V. I. Leo, M. Low, T. W. Mak, X. Zhang, P. T. Reilly, A. Lewin, Ed. Targeted ANP32E mutant mice do not demonstrate obvious movement defects, PLoS One 8, e63815 (2013).

42. P. Wong, C. C. R. Chang, C. E. Marx, M. G. Caron, W. C. Wetsel, X. Zhang, K. Hashimoto, Ed. Pregnenolone rescues schizophrenia-like behavior in dopamine transporter knockout mice, PLoS One 7, e51455 (2012).

43. V. A. Narkar, M. Downes, R. T. Yu, E. Embler, Y.-X. Wang, E. Banayo, M. M. Mihaylova, M. C. Nelson, Y. Zou, H. Juguilon, H. Kang, R. J. Shaw, R. M. Evans, AMPK and PPARdelta agonists are exercise mimetics, Cell 134, 405-415 (2008).

44. K. Nagy, M. Tóth, P. Major, G. Patay, G. Egri, J. Häggkvist, A. Varrone, L. Farde, C. Halldin, B. Gulyás, Performance evaluation of the small-animal nanoScan PET/MRI system, J. Nucl. Med. 54, 1825-1832 (2013).

45. D. L. Topping, P. M. Clifton, Short-chain fatty acids and human colonic function: 
roles of resistant starch and nonstarch polysaccharides, Physiol. Rev. 81, 1031-1064 (2001).

46. M. Watanabe, S. M. Houten, C. Mataki, M. A. Christoffolete, B. W. Kim, H. Sato, N. Messaddeq, J. W. Harney, O. Ezaki, T. Kodama, K. Schoonjans, A. C. Bianco, J. Auwerx, Bile acids induce energy expenditure by promoting intracellular thyroid hormone activation, Nature 439, 484-489 (2006).

47. P. M. Smith, M. R. Howitt, N. Panikov, M. Michaud, C. A. Gallini, M. Bohlooly-Y, J. N. Glickman, W. S. Garrett, The microbial metabolites, short-chain fatty acids, regulate colonic Treg cell homeostasis, Science 341, 569-573 (2013).

48. A. C. Dona, B. Jiménez, H. Schäfer, E. Humpfer, M. Spraul, M. R. Lewis, J. T. M. Pearce, E. Holmes, J. C. Lindon, J. K. Nicholson, Precision high-throughput proton NMR spectroscopy of human urine, serum, and plasma for large-scale metabolic phenotyping, Anal. Chem. 86, 9887-894 (2014).

49. J. M. Posma, S. L. Robinette, E. Holmes, J. K. Nicholson, MetaboNetworks, an interactive Matlab-based toolbox for creating, customizing and exploring sub-networks from KEGG, Bioinformatics 30, 893-895 (2014).

50. S. Masuda, T. Hayashi, T. Hashimoto, S. Taguchi, Correlation of dystrophinglycoprotein complex and focal adhesion complex with myosin heavy chain isoforms in rat skeletal muscle, Acta Physiol. (Oxf). 195, 483-494 (2009).

Acknowledgments: We thank Velmurugesan Arulampalam and Tak Mak for helpful suggestions. We also thank Teo Wei Ling, Arlaine Anne Amoyo-Brion, Seow Shih Wee, Peiyan Wong, Annika Samuelsson, and Johanna Aspsäter for technical assistance. We thank the Center for Germ Free Research (CFGR), Karolinska Institutet, Stockholm, Sweden; Mouse Metabolic Evaluation Facility (MEF), Genomics Technology Facility (GTF), Center for Integrative Genomics (CIG), Lausanne, Switzerland; and Duke-NUS Behavioral Phenotyping Core Facility, Duke-NUS vivarium, and SingHealth Experimental Medical Centre for their technical help and support. 
Funding: This project was supported by grants from the 7th European Union (EU) program TORNADO (SP and WW); the Swiss National Science Foundation (SNSF) (WW and JA); Fondation Suisse de Recherche sur les Maladies Musculaires and Fondation Marcel Levaillant (JA); Vetenskapsrådet, EU project "Molecular Targets Open for Regulation by the Gut Flora-New Avenues for Improved Diet to Optimize European Health," Hjärnfonden, Mérieux Institute; Singapore Millennium Foundation; and the startup grant from the Lee Kong Chian School of Medicine, Nanyang Technological University (SP and WW). We also thank the SNSF (P300P3_151157) and European Society for Clinical Nutrition and Metabolism (ESPEN) for providing grant support to SL. IGP is supported by a NIHR career development research fellowship (NIHR-CDF2017-10-032). MJB was supported in part by NIH grant \# R01-DK090989. JMP is supported by a Rutherford Fund Fellowship at Health Data Research UK (\# $\mathrm{MR} / \mathrm{S} 004033 / 1)$. EH is supported by the UK Dementia Research Institute (UK-DRI) at Imperial College London.

The views expressed are those of the authors and not necessarily those of the UK National Health Service (NHS), the NIHR, or the UK Department of Health.

Author contributions: SL, WW, and SP conceived and designed the project.

SL performed experiments on the entire characterization of the atrophy phenotype and metabolic characterization of the different experimental groups in skeletal muscle and liver through western blot and qPCRs; SL performed glucocorticoid induced atrophy in C2C12 cell lines; IGP performed the NMR spectroscopic experiments; JMP prepared the metabonetwork map; SL, HK and MMR performed the NMJ experiments; HK and MMR performed SCFA treatment; KM performed the weight test: JS and KM performed the open field activity test; PK performed the AMPK western blot; LMC performed antibiotic treatment; SL and HZ performed cellular respiration in C2C12 myotubes; PP performed PET-MRI experiment; CM performed histology and histochemical assay.

SL analyzed qPCR, WB and histological datasets of tissues from different experimental groups; SL analyzed antibiotic treatment and glucocorticoid induced atrophy in $\mathrm{C} 2 \mathrm{C} 12$ myotubes dataset; IGP and JMP analyzed NMR spectroscopic dataset; HZ and MMR analyzed SCFA treatment dataset; PP analyzed the PET-MRI data; SL and HZ analyzed 
data on cellular respiration in $\mathrm{C} 2 \mathrm{C} 12$ myotubes; JS and $\mathrm{KM}$ analyzed data on open field activity test. WW and SP gave input to overall analyses of the data. SL and SP wrote the manuscript with input from JA, EH, JN and WW.

Competing interests: All the authors declare no conflict of interest.

Data and Materials Availability. All data associated with this study are in the main text or supplementary materials.

\section{Figure Legends}

Fig. 1. The gut microbiota influences skeletal muscle mass and function in mice. (A) Weights of soleus, gastrocnemius, tibialis anterior (TA), quadriceps, and extensor digitorum longus (EDL) muscles from pathogen-free mice (PF), germ-free mice (GF), and germ-free mice transplanted with the gut microbiota of pathogen-free mice, referred to as conventionalized germ-free mice (C-GF). The number of mice used per experimental group: Soleus muscle (PF, $n=13 ; \mathrm{GF}, \mathrm{n}=14$; C-GF, $\mathrm{n}=10$ ); gastrocnemius muscle (PF, n=15; GF, n=14; C-GF, n=13); TA muscle (PF, n=14; GF, n=13; C-GF, $\mathrm{n}=12$ ); quadriceps muscle ( $\mathrm{PF}, \mathrm{n}=15 ; \mathrm{GF}, \mathrm{n}=14 ; \mathrm{C}-\mathrm{GF}, \mathrm{n}=13$ ); EDL muscle (PF, $\mathrm{n}=13$; GF, $\mathrm{n}=12$; C-GF, $\mathrm{n}=13$ ). (B) Changes in expression of genes encoding myosin heavy chain $(\mathrm{MyHC})$ isoforms in TA muscles of $\mathrm{PF}, \mathrm{GF}$, and C-GF mice ( $\mathrm{n}=5-10$ per group). (C) Changes in expression of Atrogin-1, Murf-1 and FoxO3 genes in TA muscles from PF ( $\mathrm{n}=7), \mathrm{GF}(\mathrm{n}=7)$, and C-GF (n=9) mice. (D) Shown are changes in the expression of genes encoding the skeletal muscle-specific transcription factors MyoD and Myogenin in TA muscle samples from PF ( $n=7)$, GF $(n=7)$, and C-GF mice $(n=9)$. (E) Shown is immunoblot analysis of protein lysates from TA muscles harvested from PF, GF, and CGF mice indicating expression of Atrogin-1 ( $\mathrm{n}=4$ mice per group), Murf-1 ( $\mathrm{n}=4$ mice per group), and phosphorylated AMPK (p-AMPK) ( $\mathrm{n}=5$ mice per group). (F) The results in 
the histogram are expressed as the ratio of relative intensity of Atrogin-1 and Murf-1 protein expression normalized to tubulin as loading control, and relative intensity of pAMPK expression relative to total AMPK expression.

Fig.2. The gut microbiota regulates branched chain amino acid (BCAA) metabolism in skeletal muscle. (A) Shown are measurements of serum corticosterone concentrations in pathogen-free mice ( $P F, n=17)$, germ-free mice $(\mathrm{GF}, \mathrm{n}=16)$, and germ-free mice transplanted with the gut microbiota of pathogen-free mice, referred to as conventionalized germ-free mice (C-GF, $\mathrm{n}=10)$. (B) Gene expression of Klf15 in TA muscles from PF ( $\mathrm{n}=7), \mathrm{GF}(\mathrm{n}=7)$, and C-GF $(\mathrm{n}=9)$ mice. (C) Changes in expression of genes involved in BCAA catabolism (Bcat2, Bckdk, Bckdh) in TA muscles of PF, GF, and C-GF mice ( $\mathrm{n}=6,7$ and 9, respectively). (D) Changes in the expression of the genes Igfl and Igf binding proteins (Igfbps) in tibialis anterior muscle of PF mice ( $\mathrm{n}=7)$, GF mice $(\mathrm{n}=7)$ and C-GF mice $(\mathrm{n}=9)$. Data are expressed as mean \pm SEM. Data are analyzed using ANOVA followed by Tukey's post-hoc test and were considered statistically significant at $* \mathrm{P}<0.05, * * \mathrm{P}<0.01$, and $* * * \mathrm{P}<0.001$ between indicated groups.

\section{Fig. 3. The gut microbiota influences the oxidative capacity of mouse skeletal} muscle. (A)Representative images of TA muscle sections from pathogen-free (PF), germ-free (GF), and germ-free mice transplanted with the gut microbiota of pathogenfree mice, referred to as conventionalized germ-free mice (C-GF) mice stained for Succinic Dehydrogenase enzyme (SDH) (Scale bar, 100um). (B) Gene expression of $S d h$ in tibialis anterior (TA) muscle from $\mathrm{PF}(\mathrm{n}=7), \mathrm{GF}(\mathrm{n}=7)$, and C-GF mice ( $\mathrm{n}=9)$. (C) Quantitative analysis of the ratio of mitochondrial DNA copy number (mtDNA) to nuclear DNA in gastrocnemius muscles from $\mathrm{PF}(\mathrm{n}=4), \mathrm{GF}(\mathrm{n}=5)$, and C-GF mice $(\mathrm{n}=5)$. (D, E) Changes in expression of PGC-1 $\alpha$ and Tfam genes (D), and CoxVa, CoxVIIb, Cyt c genes $(\mathbf{E})$ in TA muscles of PF $(n=6), G F(n=7)$, and C-GF mice $(n=9)$. (F) Changes in expression of genes involved in glucose metabolism $(P f k, P k, L d h$ and $P d h)$ in TA muscles of PF ( $n=6), G F(n=7)$, and C-GF mice ( $n=9)$. (G) Changes in expression of genes involved in the fatty acid oxidation pathway (Lcad, Mcad and Cpt1b) in TA 
muscles of PF (n=7), GF (n=7), and C-GF ( $\mathrm{n}=9)$ mice. (H) Amount of glycogen in quadriceps muscles of $\mathrm{PF}(\mathrm{n}=4), \mathrm{GF}(\mathrm{n}=3)$, and C-GF mice $(\mathrm{n}=4)$. Data are expressed as mean \pm SEM. Statistical significance ${ }^{* * *} \mathrm{P}<0.001$ between indicated groups. All data are expressed as mean \pm SEM. Data analysed using ANOVA followed by Tukey's post-hoc test and were considered statistically significant at ${ }^{*} \mathrm{P}<0.05$, ${ }^{*} * \mathrm{P}<0.01$, and $* * * \mathrm{P}<0.001$ between indicated groups.

Figure 4. Metabolite analysis of mouse muscle, liver and serum. (A-C) Shown is the average ${ }^{1} \mathrm{H}$ NMR spectrum of hydrophilic phase after Folch extraction for 25 metabolites. The ${ }^{1} \mathrm{H}$ NMR spectrum is shown for (A) gastrocnemius muscle from pathogen-free mice $(\mathrm{PF}, \mathrm{n}=8)$, germ-free mice $(\mathrm{GF}, \mathrm{n}=8)$ and germ-free mice transplanted with the gut microbiota of pathogen-free mice, referred to as conventionalized germ-free mice (C-GF $\mathrm{n}=10)(\mathbf{B})$ liver tissue from PF mice $(\mathrm{n}=7)$, GF mice $(\mathrm{n}=8)$ and C-GF mice $(\mathrm{n}=10) ;(\mathbf{C})$ serum from PF mice $(n=8)$, GF mice $(n=8)$, C-GF mice $(n=9)$. Key: 1, taurocholic acid; 2, bile acids; 3, lipid (Low Density Lipoproteins; LDL); 4, lipid (Very Low Density Lipoproteins; VLDL); 5, leucine; 6, 3-hydroxybutyrate; 7, alanine; 8, acetate; 9, glutamine; 10, glutamate; 11, pyruvate; 12, glutathione; 13, hypotaurine; 14, dimethylamine; 15 , sarcosine; 16 , trimethylamine; 17, dimethylglycine; 18 , unknown (83.11) (s); 19, choline; 20, glycerophosphorylcholine; 21, taurine; 22, betaine; 23, glycine; 24, unknown (83.59) (d); 25, unknown (83.71) (s). Metabolites labelled in red were found in higher concentrations in GF mice compared to C-GF or PF mice; metabolites in blue were found in lower concentrations in GF mice compared to C-GF or PF mice. (Key: s, singlet; d, doublet, dd, doublet of doublets; t, triplet; m, multiplet). 
Fig. 5. Effect of the gut microbiota on mouse neuromuscular junction (NMJ) proteins. (A) Changes in levels of expression of genes encoding acetylcholine receptors (Chrn) in tibialis anterior muscle of PF, GF and C-GF mice including Chrnal (PF, $\mathrm{n}=7$; GF, n=7; C-GF, n=9), Chrnb (PF, n=7; GF, n=7; C-GF, n=8), Chrnd (PF, n=7; GF, n=7; C-GF, n=7) and Chrne (PF: n=7; GF: n=7; C-GF: n=7). (B) Changes in levels of gene expression in tibialis anterior muscle of $\mathrm{PF}$, GF and $\mathrm{C}-\mathrm{GF}$ mice of receptor-associated protein of the synapse (Rapsyn; PF, n=7; GF, n=7; C-GF, n=9), low-density lipoprotein receptor-related protein 4 (Lrp4), and agrin (Agrn) $(\mathrm{PF}, \mathrm{n}=7 ; \mathrm{GF}, \mathrm{n}=7 ; \mathrm{C}-\mathrm{GF}, \mathrm{n}=9)$. (C) Changes in levels of the expression of the gene encoding fast-twitch troponin (Tnn) in tibialis anterior (TA) muscles of PF mice $(n=6)$; GF mice $(n=7)$, C-GF mice $(n=9)$. (D) Analysis of hindlimb muscle grip strength using the weights test in PF, GF and C-GF mice ( $n=6$ per group). (E-F). Shown is the spontaneous activity of GF, PF and C-GF mice in the open field test measured by cumulative distance traveled (E) and cumulative vertical activities (F). PF mice $(n=8)$, GF mice $(n=9)$, and C-GF mice $(n=8)$ were monitored over a 2 hour period. All data are expressed as mean \pm SEM. Data were analysed using ANOVA followed by Tukey's post-hoc test and were considered statistically significant at $* \mathrm{P}<0.05, * * \mathrm{P}<0.01$, and $* * * \mathrm{P}<0.001$ between indicated groups.

Fig. 5. Bacterial metabolites influence skeletal muscle function in mice. (A) Effect of short chain fatty acids (SCFAs) on dexamethasone (Dex)-induced muscle atrophy in C2C12 mouse myotubes in vitro. Differentiated myotubes were treated with a cocktail of SCFAs $(10 \mathrm{mM})$ in the presence or absence of dexamethasone $(1 \mathrm{mM})$ for 24 hours, and relative gene expression of Atrogin-1 and Murf-1 was analyzed ( $\mathrm{n}=3$ per group). For the control group, myotubes were treated with solvent $(0.1 \%$ DMSO) only as vehicle. $(\mathbf{B}, \mathbf{C})$ Effect of SCFAs on the expression of genes encoding mitochondrial proteins in $\mathrm{C} 2 \mathrm{C} 12$ myotubes in vitro. Differentiated myotubes were treated with a cocktail of SCFAs (10 $\mathrm{mM}$ ) for 24 hours and relative gene expression of (B) $P G C-1 \alpha$ and Tfam ( $\mathrm{n}=3$ per group), and (C) CoxVa, CoxVIIb, and Cyt $c$ were analyzed ( $\mathrm{n}=3$ per group). (D) Relative weights of soleus, gastrocnemius, tibialis anterior (TA), quadriceps, and EDL muscles from GF 
mice either untreated or treated with SCFAs (GF+SCFAs) ( $\mathrm{n}=6$ mice per group). (E-G) Changes in gene expression of Atrogin-1,Murf-1 and MyoD (E ), PGC-1 $\alpha$ and Tfam (F), and CoxVa, CoxVIIb, and Cyt c (G) in tibialis anterior (TA) muscles from untreated and SCFA-treated GF mice ( $\mathrm{n}=6$ per group). (H) Analysis of hindlimb muscle grip strength in untreated and SCFA-treated GF mice using the weights test ( $\mathrm{n}=6$ per group). (I) Relative gene expression of Rapsyn, Lrp4, and Agrn in TA muscles from untreated and SCFA-treated GF mice ( $\mathrm{n}=6$ mice per group). All data are expressed as mean $\pm \mathrm{SEM}$. For panel A, data were analysed using ANOVA followed by Tukey's post-hoc test. For all other panels, data were analysed using two-tailed Student's t-test. Data were considered statistically significant at $* \mathrm{P}<0.05, * * \mathrm{P}<0.01$, and $* * * \mathrm{P}<0.001$ between indicated groups.

Table.1. Differences in metabolite concentrations between GF, PF and C-GF mice.

\begin{tabular}{|c|c|c|c|c|}
\hline Tissue & $\begin{array}{l}\text { Metabolites lower in } \\
\text { GF mice }\end{array}$ & $\begin{array}{r}\text { Chemical } \\
\text { shift (ppm) }\end{array}$ & $\begin{array}{c}\text { Metabolites } \\
\text { higher in GF } \\
\text { mice }\end{array}$ & $\begin{array}{c}\text { Chemical shift } \\
\text { (ppm) }\end{array}$ \\
\hline \multirow[b]{2}{*}{$\begin{array}{l}\text { Skeletal } \\
\text { muscle }\end{array}$} & Unknown & $3.11(\mathrm{~s})$ & Glycine & $3.57(\mathrm{~s})$ \\
\hline & & & Alanine & $1.48(\mathrm{~d})$ \\
\hline \multirow{7}{*}{ Liver } & Unknown & $3.11(\mathrm{~s})$ & Taurine & $3.43(\mathrm{t}) ; 3.27(\mathrm{t})$ \\
\hline & Glutamine & $\begin{array}{l}2.15(\mathrm{~m}) \\
2.44(\mathrm{~m})\end{array}$ & $\begin{array}{l}\text { Taurocholic } \\
\text { acid }\end{array}$ & $0.70(\mathrm{~s})$ \\
\hline & Betaine & $\begin{array}{l}3.27(\mathrm{~s}) \\
3.90(\mathrm{~s})\end{array}$ & Hypotaurine & $2.65(\mathrm{t})$ \\
\hline & Alanine & 1.48 (d) & Dimethylamine & $2.72(\mathrm{~s})$ \\
\hline & Leucine & $0.96(t)$ & Sarcosine & $2.74(\mathrm{~s})$ \\
\hline & Valine & $\begin{array}{l}0.99(\mathrm{~d}) \\
1.05(\mathrm{~d})\end{array}$ & & \\
\hline & Pyruvate & $2.39(\mathrm{~s})$ & & \\
\hline
\end{tabular}




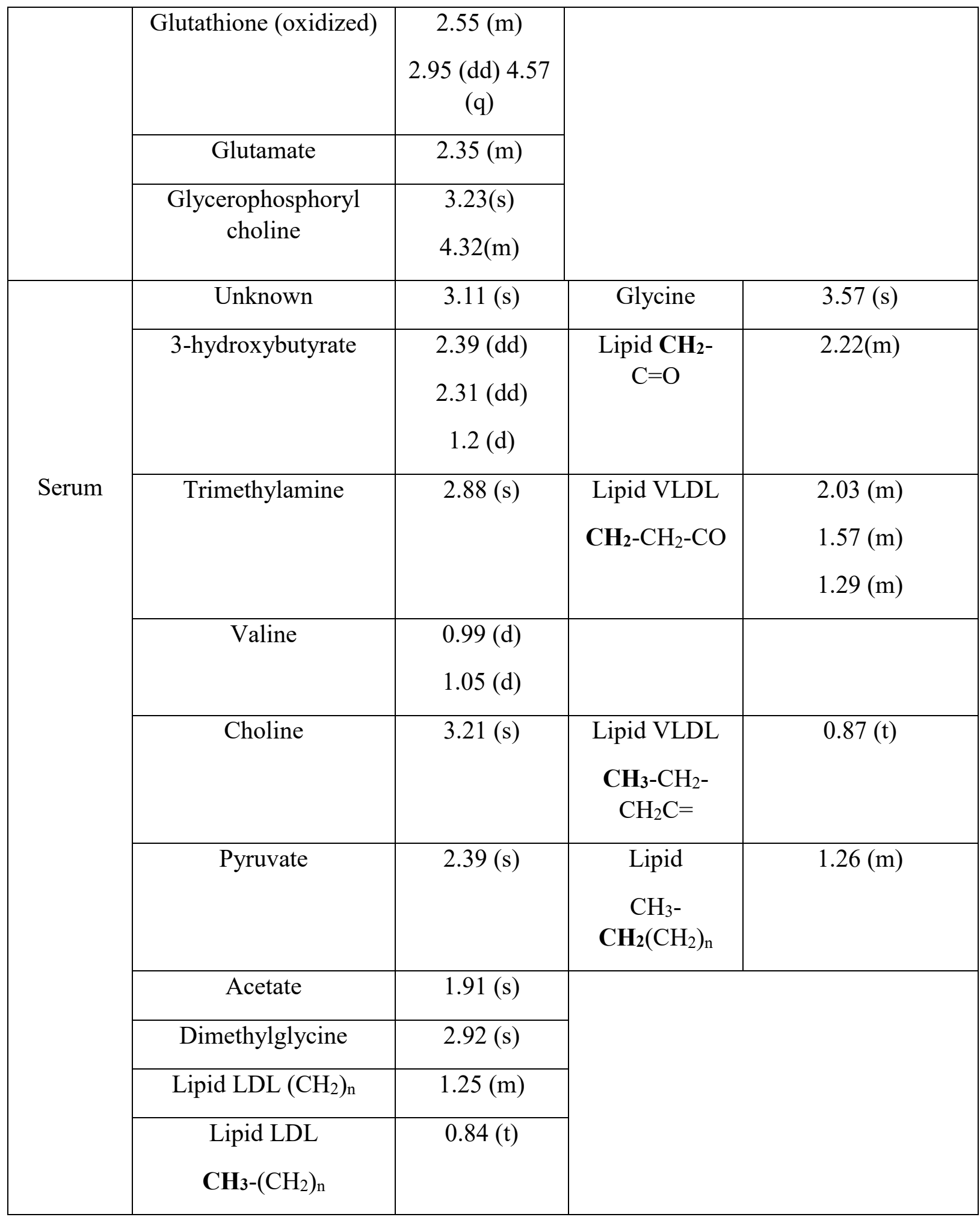

Key: s, singlet; d, doublet, dd, doublet of doublets; $t$, triplet; m, multiplet 